\title{
ЭКВИВАЛЕНТНОСТЬ СУЩЕСТВОВАНИЯ НЕСОПРЯЖЕННЫХ И НЕИЗОМОРФНЫХ ХОЛЛОВЫХ $\pi$-ПОДГРУПП ${ }^{1}$
}

\author{
В. Го, А. А. Бутурлакин, Д. О. Ревин
}

\begin{abstract}
Пусть $\pi$ - некоторое множество простых чисел. Подгруппа $H$ конечной группы $G$ называется холловой $\pi$-подгруппой, если любой простой делитель порядка $|H|$ подгруппы $H$ принадлежит $\pi$, а индекс $|G: H|$ не делится на числа из $\pi$. Знаменитая теорема Холла утверждает, что разрешимая конечная группа всегда содержит холлову $\pi$-подгруппу, и любые две холловы $\pi$-подгруппы в такой группе сопряжены. Справедливо обращение теоремы Холла: для любой неразрешимой группы $G$ можно указать множество $\pi$ такое, что $G$ не содержит холловых $\pi$-подгрупп. Тем не менее, холловы $\pi$-подгруппы могут существовать и в неразрешимой группе. Известны примеры множеств $\pi$ таких, что в любой конечной группе, содержащей холлову $\pi$-подгруппу, все холловы $\pi$-подгруппы сопряжены (и, как следствие, изоморфны). Так в 1987 г. $\Phi$. Гросс показал, что этим свойством обладает любое множество $\pi$ нечетных простых чисел. Наряду с этим, в неразрешимых группах для некоторых $\pi$ холловы $\pi$-подгруппы могут быть несопряженными, но изоморфными (скажем, в $P S L_{2}(7)$ для $\pi=\{2,3\}$ ), и даже неизоморфными (в $P S L_{2}(11)$ для $\pi=\{2,3\}$ ). В работе доказано, что для множества $\pi$ существование конечной группы с несопряженными холловыми $\pi$-подгруппами влечет существование группы с неизоморфными холловыми $\pi$-подгруппами. Обратное утверждение очевидно.
\end{abstract}

Ключевые слова: холлова $\pi$-подгруппа, свойство $\mathscr{C}_{\pi}$, сопряженные подгруппы.

W. Guo, A. A. Buturlakin, D. O. Revin. Equivalence of the existence of nonconjugate and nonisomorphic Hall $\pi$-subgroups.

Let $\pi$ be some set of primes. A subgroup $H$ of a finite group $G$ is called a Hall $\pi$-subgroup if any prime divisor of the order $|H|$ of the subgroup $H$ belongs to $\pi$ and the index $|G: H|$ is not a multiple of any number in $\pi$. The famous Hall theorem states that a solvable finite group always contains a Hall $\pi$-subgroup and any two Hall $\pi$-subgroups of such group are conjugate. The converse of the Hall theorem is also true: for any nonsolvable group $G$, there exists a set $\pi$ such that $G$ does not contain Hall $\pi$-subgroups. Nevertheless, Hall $\pi$-subgroups may exist in a nonsolvable group. There are examples of sets $\pi$ such that, in any finite group containing a Hall $\pi$-subgroup, all Hall $\pi$-subgroups are conjugate (and, as a consequence, are isomorphic). In $1987 \mathrm{~F}$. Gross showed that any set $\pi$ of odd primes has this property. In addition, in nonsolvable groups for some sets $\pi$, Hall $\pi$-subgroups can be nonconjugate but isomorphic (say, in $P S L_{2}(7)$ for $\pi=\{2,3\}$ ) and even nonisomorphic (in $P S L_{2}$ (11) for $\pi=\{2,3\}$ ). We prove that the existence of a finite group with nonconjugate Hall $\pi$-subgroups for a set $\pi$ implies the existence of a group with nonisomorphic Hall $\pi$-subgroups. The converse statement is obvious.

Keywords: Hall $\pi$-subgroup, $\mathscr{C}_{\pi}$ condition, conjugate subgroups.

MSC: 20D20

DOI: $10.21538 / 0134-4889-2018-24-3-43-50$

Александру Алексеевичу Махневу к 65-летию

\section{1. Введение}

Мы рассматриваем только конечные группы, и в связи с этим термин "группа" употребляется нами в значении "конечная группа". Через $\pi$ обозначается некоторое множество простых чисел, а через $\pi^{\prime}$ - множество всех простых чисел, не лежащих в $\pi$. Подгруппа $H$ группы $G$

\footnotetext{
${ }^{1}$ Первый автор поддержан Национальным естественнонаучным фондом Китая (NNSF), грант № 11771409. Второй автор поддержан программой фундаментальных научных исследований СО PAH No I.1.1., проект № 0314-2016-0001. Третий автор поддержан Стипендиальной инициативой Президента Китайской академии наук (PIFI), грант № 2016VMA078, и Российским фондом фундаментальных исследований, грант No 17-51-45025.
} 
называется холловой $\pi$-подгруппой, если любой простой делитель порядка подгруппы $H$ принадлежит $\pi$ (т. е. $H$ является $\pi$-группой), в то время как $|G: H|$ не делится на числа из $\pi$.

В соответствии с обозначениями $\Phi$. Холла [1] будем говорить, что группа $G$ обладает свой$c m в о м \mathscr{E}_{\pi}$, если в $G$ имеется хотя бы одна холлова $\pi$-подгруппа. Если в группе $G$ со свойством $\mathscr{E}_{\pi}$ любые две холловы $\pi$-подгруппы сопряжены, то будем говорить, что $G$ обладает свойством $\mathscr{C}_{\pi}$. Группу со свойством $\mathscr{E}_{\pi}$ или $\mathscr{C}_{\pi}$ будем называть также $\mathscr{E}_{\pi}$ - или $\mathscr{C}_{\pi}$-группой. Символы $\mathscr{E}_{\pi}$ и $\mathscr{C}_{\pi}$ будут использоваться также для обозначения классов всех $\mathscr{E}_{\pi^{-}}$и $\mathscr{C}_{\pi}$-групп соответственно. По аналогии с данными обозначениями пусть $\mathscr{I}_{\pi}-$ класс $\mathscr{E}_{\pi}$-групп, в которых любые две холловы $\pi$-подгруппы изоморфны. Ясно, что

$$
\mathscr{C}_{\pi} \subseteq \mathscr{I}_{\pi} \subseteq \mathscr{E}_{\pi} .
$$

Существуют множества $\pi$, для которых $\mathscr{E}_{\pi}=\mathscr{C}_{\pi}$. Такими будут множество всех простых чисел, пустое множество и, ввиду теоремы Силова, одноэлементные множества. Ф. Гросс [2] показал также, что этим свойством обладает любое множество нечетных простых чисел. В то же время существуют множества $\pi$ такие, что $\mathscr{E}_{\pi} \neq \mathscr{C}_{\pi}$. Таким является множество $\pi=\{2,3\}$, поскольку группа $G L_{3}(2)$ обладает несопряженными (хотя и изоморфными) холловыми $\pi$ подгруппами: ими будут стабилизаторы прямой и плоскости естественного 3-мерного модуля над полем из двух элементов.

Пусть $\mathscr{E}_{\pi} \neq \mathscr{C}_{\pi}$. В данной работе мы покажем, что в разности $\mathscr{E}_{\pi} \backslash \mathscr{C}_{\pi}$ всегда найдется группа, содержащая неизоморфные холловы $\pi$-подгруппы.

Теорема. Для любого множества $\pi$ простых чисел если $\mathscr{E}_{\pi} \neq \mathscr{C}_{\pi}$, то $\mathscr{E}_{\pi} \neq \mathscr{I}_{\pi}$.

В работах [3;4] данный результат был доказан в случае, когда $\pi=p^{\prime}$ для некоторого простого числа $p$. Более точно, обозначим через $\mathscr{A}_{\pi}$ класс $\mathscr{E}_{\pi}$-групп $G$ таких, что любые две холловы $\pi$-подгруппы из $G$ сопряжены элементом из $\operatorname{Aut}(G)$. Очевидно, что цепочку включений (1.1) можно уплотнить до следующей:

$$
\mathscr{C}_{\pi} \subseteq \mathscr{A}_{\pi} \subseteq \mathscr{I}_{\pi} \subseteq \mathscr{E}_{\pi} .
$$

Пусть $\pi=p^{\prime}$. М. Н. Нестеров [3] показал, что в этом случае либо все включения в цепочке (1.2) одновременно строгие, либо все являются равенствами. Более того, показано, что для данного простого числа $p$ вопрос о совпадении классов $\mathscr{E}_{p^{\prime}}$ и $\mathscr{C}_{p^{\prime}}$ сводится к вопросу о представимости какой-либо степени числа $p$ в виде

$$
p^{l}=\frac{q^{m}-1}{q-1},
$$

где $q$ - степень простого числа, а $m$ - нечетное простое число. Это сложная теоретикочисловая проблема, тесно связанная с такими известными вопросами, как гипотеза НагелляЛюнггрена [5]. Вопрос о совпадении классов $\mathscr{E}_{\pi}$ и $\mathscr{C}_{\pi}$ в общем случае, очевидно, является еще более сложной проблемой.

В свете выше сформулированной теоремы естественно задаться вопросом: если для множества $\pi$ включение $\mathscr{C}_{\pi} \subset \mathscr{E}_{\pi}$ строгое, то будет ли строгим включение $\mathscr{C}_{\pi} \subset \mathscr{I}_{\pi}$ ? Авторам неизвестен ответ на этот вопрос. Возможно, контрпримером будет множество $\{2,7\}$. Как следует из теоремы С.А. Чунихина (см. лемму 1 ), строгость включения $\mathscr{C}_{\pi} \subset \mathscr{E}_{\pi}$ эквивалентна существованию простой группы в разности $\mathscr{C}_{\pi} \backslash \mathscr{E}_{\pi}$. Если $\pi=\{2,7\}$, то любая простая группа из этой разности изоморфна некоторой группе Ри ${ }^{2} G_{2}\left(3^{2 n+1}\right)$ и обладает неизоморфными холловыми подгруппами [6, теорема 8.9]. Вопрос в том, будет ли данное утверждение верно для произвольной группы $G \in \mathscr{C}_{\{2,7\}} \backslash \mathscr{E}_{\{2,7\}}$.

Из теоремы получаем

Следствие 1. Для любого множества $\pi$ простых чисел эквивалентны следующие утверждения. 
(1) Существует группа с несопряженными холловыми $\pi$-подгруппами.

(2) Существует группа с неизоморфными холловыми л-подгруппами.

Разность классов $\mathscr{E}_{\pi} \backslash \mathscr{C}_{\pi}$, если только она непуста для данного множества $\pi$, всегда содержит примеры групп с достаточно "патологическими" холловыми $\pi$-подгруппами, поведение которых резко отличается от привычного поведения холловых подгрупп в разрешимых группах. Свойства групп в этой разности исследовались в работах [7-10], см. также обзор [6] и монографию [11, гл. 2]. Основной упор в этих работах сделан на изучении пронормальности холловых подгрупп.

Напомним, что согласно определению $\Phi$. Холла [12] подгруппа $H$ группы $G$ называется $n p o-$ нормальной, если для любого элемента $g \in G$ подгруппы $H$ и $H^{g}$ сопряжены в $\left\langle H, H^{g}\right\rangle$. Хотя холлова подгруппа, вообще говоря, непронормальна [7, теорема 3], именно в терминах пронормальности удается сформулировать наиболее сильные общие свойства холловых подгрупп в произвольных группах. Так, оказывается, что холловы $\pi$-подгруппы пронормальны в простых группах [13, теорема 1] и в $\mathscr{C}_{\pi}$-группах [7, теорема 2] и, более того, существование в конечной группе $G$ холловой $\pi$-подгруппы (т. е. $G \in \mathscr{E}_{\pi}$ ) означает существование в $G$ и в каждой ее нормальной подгруппе холловой $\pi$-подгруппы, пронормальной в $G$ [14, теорема 1 и следствие 2]. Формальным следствием ${ }^{2}$ этого утверждения является аналог аргумента Фраттини для холловых подгрупп [15, теорема 1]: если $G \in \mathscr{E}_{\pi}$, то в любой нормалъной подгруппе $A$ группъ $G$ найдется холлова $\pi$-подгруппа $H$ такал, что $G=A N_{G}(H)$. С другой стороны, существование для данного множества $\pi$ групп с несопряженными холловыми $\pi$-подгруппами эквивалентно существованию группы с непронормальными холловыми $\pi$-подгруппами [10, предложение 1]. М.Н.Нестеров [8] нашел примеры холловых подгрупп, непронормальных не только во всей группе, но даже в своем нормальном замыкании. Более того, оказалось [9, теорема], что для данного $\pi$ существование групп с несопряженными холловыми $\pi$-подгруппами эквивалентно не только существованию групп с непронормальными холловыми $\pi$-подгруппами, но и существованию групп, в которых имеются холловы $\pi$-подгруппы, не пронормальные в своем нормальном замыкании.

Объединяя последний результат с результатами данной работы, получаем

Следствие 2. Для любого множества $\pi$ простых чисел эквивалентны следующие утверждения.

(1) Существует группа с несопряженными холловыми $\pi$-подгруппами.

(2) Существует простая группа с несопряженными холловыми т-подгруппами.

(3) Существует группа с холловыми п-подгруппами, не сопряженными в группе автоморфизмов.

(4) Существует группа с неизоморфными холловыми л-подгруппами.

(5) Существует группа с непронормальной холловой $\pi$-подгруппой.

(6) Существует группа с холловой $\pi$-подгруппой, не пронормальной в своем нормальном замыкании.

\footnotetext{
23десь речь идет именно о формальном следствии, поскольку в доказательстве результатов из [14] используются результаты работы [15]. Другие многочисленные утверждения, вытекающие из [14, теорема 1], можно найти в обзоре [16].
} 


\section{2. Предварительные результаты}

Лемма 1 (теорема С.А.Чунихина) [17, гл. 5, (3.12); 11, гл. 2, лемма 2.4]. Eсли $N \unlhd G u N$, $G / N \in \mathscr{C}_{\pi}, m o G \in \mathscr{C}_{\pi}$.

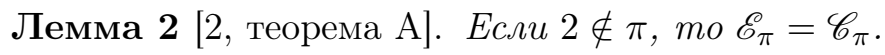

Лемма 3. Пусть $3 \notin \pi u G \in \mathscr{E}_{\pi} \backslash \mathscr{C}_{\pi}-$ простая группа. Тогда справедливъ следующие утверждения.

(1) $G \cong{ }^{2} G_{2}(q)$ для некоторого $q=3^{2 n+1}$.

(2) $\{2,7\}=\pi \cap \pi(G)$.

(3) $G$ содержит ровно два класса сопряженных холловых $\pi$-подгрупп. Подгруппы в одном из классов являются группами Фробениуса порлдка 56 с ядром порлдка 8. Подгруппы другого класса содержат нормальную силовскую 7-подгруппу. В частности, несопряженные холловы л-подгруппы в $G$ неизоморфны.

Д о к а з а т е л ь с т в о. По лемме 2 можно считать, что $2 \in \pi$. Из [6, теоремы 8.1 и $8.2]$ следует, что $G$ - группа лиева типа. Обозначим через $p$ характеристику поля определения группы $G$. Предположим, что $p \in \pi$. Тогда $[6$, теорема 8.3$]$ влечет, что любая холлова $\pi$-подгруппа содержится в подгруппе Бореля. Поскольку все подгруппы Бореля сопряжены и разрешимы, из теоремы Холла следует, что все холловы $\pi$-подгруппы в $G$ сопряжены; противоречие. Таким образом, $p \notin \pi$. Теперь заключение леммы прямо следует из [6, теорема 8.9].

Лемма 4. Пусть $G=G L_{3}(2)$ u $V$ - естественный $\mathbb{F}_{2} G$-модуль. Пусть $H$ и $K$ соответственно - стабилизаторы в $G$ некоторых прямой и плоскости из пространства $V$. Обозначим через $G^{*}$ естественное полупрямое произведение $V G$. Тогда

(1) $H$ и $K$ - несопряженные холловы $\{2,3\}$-подгруппы в $G$.

(2) $H^{*}=V H$ и $K^{*}=V K-$ холловы $\{2,3\}$-подгруппь в $G^{*}$.

(3) $Z\left(H^{*}\right) \neq 1$, в то время как $Z\left(K^{*}\right)=1$. В частности, $H^{*}$ и $K^{*}$ неизоморфны.

Д о к а з а т е л ь с т в о. Утверждения леммы доказаны в $[4$, с. 415, 416] при построении примера группы с неизоморфными р-дополнениями.

Лемма 5 [7, следствие 4; 8, следствие $1 ; 9$, теорема; 10, предложение 1]. Для любого множества $\pi$ простых чисел эквивалентны следующие утверждения.

(1) Существует группа с несопряженными холловыми $\pi$-подгруппами.

(2) Существует группа с непронормальной холловой $\pi$-подгруппой.

(3) Существует группа с холловой $\pi$-подгруппой, не пронормальной в своем нормальном замыкании. 


\section{3. Доказательство основных результатов}

Ключевым фактом в доказательстве теоремы является

Лемма 6. Пусть $G-$ транзитивная группа подстановок на некотором множестве $\Omega$, содержащем более одного элемента, и $H$ - стабилизатор точки. Пусть $K-$ подгруппа группы $G$ и $S$ - неабелева простал группа. Рассмотрим подстановочное сплетение $G^{*}=S \imath G$ и обозначим через $H^{*}$ и $K^{*}$ полные прообразы относительно естественного эпиморфизма $G^{*} \rightarrow G$ подгрупп $H$ и K. Следующие утверждения эквивалентны:

(1) $H^{*}$ и $K^{*}$ изоморфны;

(2) $H^{*}$ и $K^{*}$ сопряжены в $G^{*}$;

(3) $Н$ и $K$ сопряжены в $G$.

Д о к а з а т е л ь с т в о. Импликации $(3) \Rightarrow(2)$ и $(2) \Rightarrow(1)$ очевидны. Докажем $(1) \Rightarrow(3)$.

Пусть $X$ - база сплетения $G^{*}=S \imath G$. Тогда $X=S_{1} \times \cdots \times S_{n}$, где $S_{i} \cong S$ - и все подгруппы $S_{i}$ сопряжены в $G^{*}=G X$. Подгруппа $X$ нормализует каждую из подгрупп $S_{i}$ и, значит, лежит в ядре действия группы $G^{*}$ сопряжениями на множестве

$$
\Omega^{\prime}=\left\{S_{i} \mid i=1, \ldots, n\right\} .
$$

Мы можем пропустить соответствующий гомоморфизм $G^{*} \rightarrow \operatorname{Sym}\left(\Omega^{\prime}\right)$ через $G$ так, что будет коммутативна диаграмма

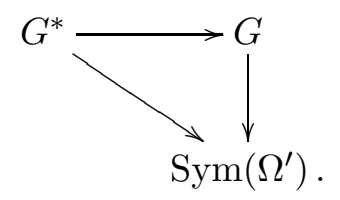

Из определения подстановочного сплетения следует, что возникающее при этом действие группы $G$ на $\Omega^{\prime}$ эквивалентно естественному действию $G$ как группы подстановок множества $\Omega$. Отсюда $C_{G^{*}}(X) \leq X$, а поскольку $S$ - неабелева простая группа, получаем

$$
C_{G^{*}}(X)=C_{X}(X)=1,
$$

и поэтому $C_{H^{*}}(X)=C_{K^{*}}(X)=1$. Это означает, что ${ }^{3}$

$$
X=\operatorname{Soc}\left(H^{*}\right)=\operatorname{Soc}\left(K^{*}\right) .
$$

Допустим в соответствии с утверждением (1), что подгруппы $H^{*}$ и $K^{*}$ изоморфны. Тогда, так как цоколь - характеристическая подгруппа, имеем

$$
H \cong H^{*} / \operatorname{Soc}\left(H^{*}\right) \cong K^{*} / \operatorname{Soc}\left(K^{*}\right) \cong K \text {. }
$$

Пусть множество $\Omega$ распадается на орбиты $\Gamma_{1}, \ldots, \Gamma_{s}$ относительно действия группы $H$ и на орбиты $\Delta_{1}, \ldots, \Delta_{t}$ относительно действия $K$. Ввиду вышесказанного, числа $s$ и $t$ соответственно равны также количеству орбит групп $H^{*}=H X$ и $K^{*}=K X$ на множестве $\Omega^{\prime}$ компонент их общего цоколя $X$. Поскольку $H^{*} \cong K^{*}$, имеем $s=t$. Из тех же соображений

$$
\left\{\left|\Gamma_{i}\right| \mid i=1, \ldots, s\right\}=\left\{\left|\Delta_{j}\right| \mid j=1, \ldots, t\right\} .
$$

\footnotetext{
${ }^{3}$ Напомним, что для конечной группы $G$ цоколем $\operatorname{Soc}(G)$ называется (характеристическая) подгруппа, порожденная всеми минимальными субнормальными подгруппами. Все такие минимальные субнормальные подгруппы группы $G$ поэлементно коммутируют, и если все они неабелевы, то цоколь группы $G$ является их прямым произведением, а сама $G$ действует сопряжениями на их множестве.
} 
Поскольку $H=G_{\alpha}$ - стабилизатор в $G$ некоторой точки $\alpha \in \Omega$, подгруппа $H$ имеет одноэлементную орбиту $\Gamma_{i}=\{\alpha\}$. Тогда и $K$ имеет одноэлементную орбиту $\Delta_{j}=\{\beta\}$ и, значит, содержится в стабилизаторе $G_{\beta}$ единственной точки $\beta \in \Delta_{j}$. Этот стабилизатор сопряжен с $H$ ввиду транзитивности группы $G$. Так как $|H|=|K|$, получаем $G_{\beta}=K$. Тем самым $H$ и $K$ сопряжены в $G$.

Д о к а з а т е л ь с т в о теоремы. Пусть $\mathscr{E}_{\pi} \neq \mathscr{C}_{\pi}$. Из леммы 1 следует, что существует простая неабелева группа $G \in \mathscr{E}_{\pi} \backslash \mathscr{C}_{\pi}$. Рассмотрим пять случаев для множества $\pi$.

С л у ч а й I: $2 \notin \pi$. Из леммы 2 следует, что $\mathscr{E}_{\pi}=\mathscr{C}_{\pi}$, поэтому данный случай можно не рассматривать.

С л у ч а й II: $3 \notin \pi$. Из леммы 3 следует, что $G \cong{ }^{2} G_{2}(q)$, и несопряженные холловы $\pi$-подгруппы группы $G$ неизоморфны. Поэтому $G \in \mathscr{E}_{\pi} \backslash \mathscr{I}_{\pi}$.

С л у ч а и III и IV: $2,3,5 \in \pi$ или $2,3,7 \in \pi$. Пусть $H$ и $K-$ несопряженные холловы $\pi$-подгруппы в $G$. Ввиду простоты группу $G$ можно рассматривать как транзитивную группу подстановок, в которой подгруппа $H$ будет стабилизатором точки. Рассмотрим подстановочное сплетение $G^{*}=S \imath G$, где $S=A_{5}$, если $2,3,5 \in \pi$, или $S=G L_{3}(2)$, если $2,3,7 \in \pi$, и обозначим через $H^{*}$ и $K^{*}$ полные прообразы относительно естественного эпиморфизма $G^{*} \rightarrow G$ подгрупп $H$ и $K$. Так как $S-\pi$-группа, база сплетения $G^{*}=S \imath G$ также является $\pi$-группой, и поэтому $H^{*}$ и $K^{*}$ - холловы $\pi$-подгруппы в $G^{*}$. Из несопряженности подгрупп $H$ и $K$ в группе $G$ и леммы 6 следует, что $H^{*}$ и $K^{*}$ неизоморфны. Таким образом, $G^{*} \in \mathscr{E}_{\pi} \backslash \mathscr{I}_{\pi}$.

С л у ч а й $\mathrm{V}: 2,3, \in \pi, 5,7 \notin \pi$. Пусть $G=G L_{3}(2)$ и $V$ - естественный $\mathbb{F}_{2} G$-модуль. Пусть $H$ и $K$ - стабилизаторы в $G$ некоторых прямой и плоскости из пространства $V$. Тогда $H$ и $K$ - несопряженные холловы $\pi$-подгруппы в $G$. В естественном произведении $G^{*}=V G$ подгруппы $H^{*}=V H$ и $K^{*}=V K$ также будут холловыми $\pi$-подгруппами и $H^{*} \nsucceq K^{*}$ по лемме 4. Поэтому $G^{*} \in \mathscr{E}_{\pi} \backslash \mathscr{I}_{\pi}$.

Д о к а з а т е л ь с т в о следствия 1. Импликация (2) $\Rightarrow(1)$ очевидна. Импликация $(1) \Rightarrow(2)$ доказана в теореме.

Д о к а з а т е л ь с т в о следствия 2. Утверждения (1) и (2) эквивалентны по теореме Чунихина (лемма 1). Эквивалентность утверждений (1), (3) и (4) установлена в следствии 1. Эквивалентность утверждений (1), (5) и (6) составляет утверждение леммы 5.

\section{СПИСОК ЛИТЕРАТУРЫ}

1. Hall P. Theorems like Sylow's // Proc. London Math. Soc. 1956. Vol. s3-6, no. 22. P. 286-304. doi: $10.1112 / \mathrm{plms} / \mathrm{s} 3-6.2 .286$.

2. Gross F. Conjugacy of odd order Hall subgroups // Bull. London Math. Soc. 1987. Vol. 19, no. 4. P. 311-319. doi: 10.1112/blms/19.4.311.

3. Buturlakin A.A., Revin D.O. On p-complements of finite groups // Сиб. электрон. мат. изв. 2013. Vol. 10. P. 414-417.

4. Нестеров М.Н. Арифметика сопряженности $p$-дополнений // Алгебра и логика. 2015. Vol. 54, no. 1. P. 53-69. doi: 10.1007/s10469-015-9320-2 .

5. Ljunggren W. Einige Sätze über unbestimmte Gleichungen von der Form $\frac{x^{n}-1}{x-1}=y^{q} / /$ Norsk Mat. Tidsskr. 1943. Vol. 25. P. 17-20.

6. Вдовин Е.П., Ревин Д.О. Теоремы силовского типа // Успехи мат. наук. 2011. Vol. 66, № 5. Р. 3-46. doi: 10.4213/rm9440.

7. Вдовин Е.П., Ревин Д.О. О пронормальности холловых подгрупп // Сиб. мат. журн. 2013. Vol. 54, no. 1. P. 35-43. doi: 10.1134/S0037446613010035.

8. Нестеров М.Н. О пронормальности и сильной пронормальности холловых подгрупп // Сиб. мат. журн. 2017. Vol. 58, № 1. P. 165-173. doi: 10.17377/smzh.2017.58.116.

9. Вдовин Е.П., Нестеров М.Н., Ревин Д.О. О пронормальности холловых подгрупп в своем нормальном замыкании // Алгебра и логика. 2017. Vol. 56, № 6. P. 573-580.

doi: 10.17377/alglog.2017.56.603 . 
10. Го В., Ревин Д.О. О классе групп с пронормальными $\pi$-холловыми подгруппами // Сиб. мат. журн. 2014. Vol. 55, no. 3. P. 509-524. doi: 10.1134/S0037446614030033.

11. Guo W. Structure theory of canonical classes of finite groups. Berlin, Springer, 2015. 359 p. ISBN: 9783662457467.

12. Hall P. Phillip Hall lecture notes on group theory - Part 6. Cambridge: University of Cambridge, 1951-1967. Available at "Washington University Digital Gateway": http://omeka.wustl.edu/omeka/items/show/10788.

13. Вдовин Е.П., Ревин Д.О. Пронормальность холловых подгрупп в конечных простых группах // Сиб. мат. журн. 2012. Vol. 53, no. 3. P. 527-542. doi: 10.1134/S0037446612020231.

14. Вдовин Е.П., Ревин Д.О. Существование пронормальных $\pi$-холловых подгрупп в $E_{\pi}$-группах // Сиб. мат. журн. 2015. Vol. 56, no. 3. P. 481-486. doi: 10.1134/S0037446615030015.

15. Revin D.O., Vdovin E.P. Frattini argument for Hall subgroups // J. Algebra. 2014. Vol. 414. P. 95-104. doi: 10.1016/j.jalgebra.2014.04.031.

16. Guo W., Revin D.O. Pronormality and submaximal $\mathfrak{X}$-subgroups // Communications in Mathematics and Statistics. 2018. P. 1-29. Published online. doi: 10.1007/s40304-018-0154-9.

17. Suzuki M. Group theory II. N Y; Berlin; Heidelberg; Tokyo: Springer-Verlag, 1986. 624 p. ISBN: 978-3-642-86887-0.

Го Вэньбинь Поступила 7.05.2018 д-р физ.-мат. наук, профессор

Департамент математики, Университет Науки и Технологии Китая,

г. Хефей, Китай

e-mail: wbguo@ustc.edu.cn

Бутурлакин Александр Александрович

канд. физ.-мат. наук, старший науч. сотрудник

Институт математики им. С. Л. Соболева СО РАН;

Новосибирский государственный университет,

г. Новосибирск

e-mail: buturlakin@math.nsc.ru

Ревин Данила Олегович

д-р физ.-мат. наук, ведущий науч. сотрудник

Институт математики им. С.Л.Соболева СО РАН;

Новосибирский государственный университет,

г. Новосибирск;

Департамент математики, Университет Науки и Технологии Китая,

г. Хефей, Китай

e-mail: revin@math.nsc.ru

\section{REFERENCES}

1. Hall P. Theorems like Sylow's. Proc. London Math. Soc., 1956, vol. s3-6, no. 22, pp. 286-304. doi: $10.1112 / \mathrm{plms} / \mathrm{s} 3-6.2 .286$.

2. Gross F. Conjugacy of odd order Hall subgroups Bull. London Math. Soc., 1987, vol. 19, no. 4, pp. 311-319. doi: $10.1112 / \mathrm{blms} / 19.4 .311$.

3. Nesterov M.N. Arithmetic of conjugacy of p-complements. Algebra Logic, 2015, vol. 54, no. 1, pp. 36-47. doi: 10.1007/s10469-015-9320-2 .

4. Buturlakin A.A., Revin D.O. On p-complements of finite groups. Sib. Elektron. Mat. Izv., 2013, vol. 10, pp. $414-417$.

5. Ljunggren W. Some theorems on indeterminate equations of the form $\frac{x^{n}-1}{x-1}=y^{q}$. Norsk Mat. Tidsskr., 1943, vol. 25, pp. 17-20 (in Norwegian).

6. Vdovin E.P., Revin D.O. Theorems of Sylow type. Russian Math. Surveys, 2011, vol. 66, no. 5, pp. 829-870. doi: 10.1070/RM2011v066n05ABEH004762 . 
7. Vdovin E.P., Revin D.O. On the pronormality of Hall subgroups. Sib. Math. J., 2013, vol. 54, no. 1, pp. 22-28. doi: 10.1134/S0037446613010035.

8. Nesterov M.N. On pronormality and strong pronormality of Hall subgroups. Sib. Math. J., 2017, vol. 58, no. 1, pp. 128-133. doi: 10.1134/S0037446617010165.

9. Vdovin E.P., Nesterov M.N., Revin D.O. Pronormality of Hall subgroups in their normal closure. Algebra Logic, 2018, vol. 56, no. 6, pp. 451-457. doi: 10.1007/s10469-018-9467-8.

10. Guo W., Revin D.O. On the class of groups with pronormal Hall $\pi$-subgroups. Sib. Math. J., 2014, vol. 55, no. 3, pp. 415-427. doi: 10.1134/S0037446614030033.

11. Guo W. Structure theory of canonical classes of finite groups. Berlin: Springer, 2015, 359 p. ISBN: 9783662457467 .

12. Hall P. Phillip Hall lecture notes on group theory - Part 6. Cambridge: University of Cambridge, 1951-1967. Available at "Washington University Digital Gateway": http://omeka.wustl.edu/omeka/items/show/10788.

13. Vdovin E.P., Revin D.O. Pronormality of Hall subgroups in finite simple groups. Sib. Math. J., 2012, vol. 53, no. 3, pp. 419-430. doi: 10.1134/S0037446612020231.

14. Vdovin E.P., Revin D.O. The existence of pronormal $\pi$-Hall subgroups in $E_{\pi}$-groups. Sib. Math. J., 2015, vol. 56, no. 3, pp. 379-383. doi: 10.1134/S0037446615030015.

15. Revin D.O., Vdovin E.P. Frattini argument for Hall subgroups. J. Algebra, 2014, vol. 414, pp. 95-104. doi: 10.1016/j.jalgebra.2014.04.031.

16. Guo W., Revin D.O. Pronormality and submaximal $\mathfrak{X}$-subgroups. Communications in Mathematics and Statistics, 2018, pp. 1-29, published online. doi: 10.1007/s40304-018-0154-9.

17. Suzuki M. Group Theory II. N Y, Berlin, Heidelberg, Tokyo, Springer-Verlag, 1986, 624 p. ISBN: 978-3-642-86887-0 .

The paper was received by the Editorial Office on May 7, 2018.

Funding Agency: The first author is supported by the National Natural Science Foundation of China (project no. 11771409). The second author is supported by Program I.1.1 for Fundamental Research of the Siberian Branch of the Russian Academy of Sciences (project no. 0314-2016-0001). The third author is supported by CAS President's International Fellowship Initiative (project no. 2016VMA078) and by the Russian Foundation for Basic Research (project no. 17-51-45025).

Wenbin Guo, Dr. Phys.-Math. Sci, Prof., University of Science and Technology of China, Hefei, 230026 China, e-mail: wbguo@ustc.edu.cn.

Aleksandr Aleksandrovich Buturlakin, Cand. Sci (Phys.-Math.), Sobolev Institute of Mathematics, Siberian Branch of the Russian Academy of Sciences, Novosibirsk, 630090 Russia; Novosibirsk State University, Novosibirsk, 630090 Russia, e-mail: buturlakin@math.nsc.ru .

Danila Olegovich Revin, Dr. Phys.-Math. Sci, Sobolev Institute of Mathematics, Siberian Branch of the Russian Academy of Sciences, Novosibirsk, 630090 Russia; Novosibirsk State University, Novosibirsk, 630090 Russia; University of Science and Technology of China, Hefei, 230026 China, e-mail: revin@math.nsc.ru . 